\title{
Psychotherapy and Mental Health as a Psychological Science Discipline
}

\author{
Hans-Ulrich Wittchen ${ }^{a, b}$ Samia Härtling ${ }^{a}$ Jürgen Hoyer ${ }^{a}$ \\ ${ }^{a}$ Institute of Clinical Psychology and Psychotherapy, Technische Universität Dresden, Dresden, Germany; \\ ${ }^{b}$ Center of Epidemiology and Longitudinal Studies (CELOS), Technische Universität Dresden, Dresden, Germany
}

\section{Keywords}

Behaviour change - Psychology · Mental health .

Psychotherapy · Health behaviour - Mental disorders

\section{Summary}

The psychological sciences offer a large spectrum of theories, principles, and methodological approaches to understand mental health, normal and abnormal functions and behaviours, as well as mental disorders. Based on continued research progress, psychology has derived a wide range of effective interventions for behaviour change and the prevention, treatment and rehabilitation of mental disorders. Thus, psychology and clinical psychology in particular should be regarded as the 'mother' science for psychotherapy and psychotherapeutic practice. This paper provides a selective overview of the scope, strengths and gaps in psychological research to depict the advances needed to inform future research agendas on mental disorders and psychological interventions in the context of psychotherapy. Most maladaptive health behaviours and mental disorders can be conceptualised as the result of developmental dysfunctions of psychological functions and processes, and as associated neurobiological and genetic processes in interaction with behaviour and the environment. An integrative translational model, linking basic and experimental research with clinical research and population-based prospective-longitudinal studies is proposed for improving identification of critical core vulnerability and risk factors and core pathogenic mechanisms. The proposed framework is expected to allow a more stringent delineation of targeted preventive and therapeutic psychological interventions and an optimisation and better understanding of cognitive-behavioural therapies and other psychological interventions. Based on a European consultation process, a 'Science of Behaviour Change' programme with the promise of improved diagnosis, treatment and prevention of both healthrisk behaviour constellations and mental disorders is proposed.

\author{
Schlüsselwörter \\ Verhaltensänderung · Psychologie - Psychische Gesundheit . \\ Psychotherapie - Gesundheitsverhalten . \\ Psychische Störungen
}

\section{Zusammenfassung}

Psychologie als "Mutterwissenschaft» für Psychotherapie und psychische Gesundheit

Psychologie als Wissenschaft bietet ein breites Spektrum an Theorien, Grundlagen und methodischen Ansätzen, um psychische Gesundheit, normale und gestörte Funktionen und Verhaltensweisen sowie psychische Störungen zu erforschen und zu "verstehen». Auf dieser Grundlage haben sich in der Klinischen Psychologie vielfältige effektive, psychologisch begründete Interventionen für die Prävention, Behandlung und Rehabilitation von psychischen Störungen ausdifferenziert. Damit ist die Psychologie als "Mutterwissenschaft" der Psychotherapie und psychotherapeutische Praxis anzusehen. Der Beitrag versucht auf der Grundlage einer Stärken-/Schwächen-Analyse der psychologischen Forschung diejenigen Themenbereiche zu definieren, die für Erkenntnisfortschritte bei psychischen Störungen und die psychotherapeutische Interventionsforschung besonders vielversprechend sein könnten. Dysfunktionale bzw. abweichende Verhaltensweisen wie auch psychische Störungen lassen sich als entwicklungsbezogene Störungen psychologischer Funktionen und Prozesse und damit assoziierter neurobiologischer und genetischer Prozesse konzeptualisieren. Für eine verbesserte Identifikation von zentralen Vulnerabilitäts- und Risikofaktoren sowie ätiologisch relevanten Schlüsselprozessen wird ein integratives translationales Modell vorgeschlagen, welches die Grundlagen- und experimentelle Forschung mit klinischer Forschung, Translation und Public-health-Implikationen verknüpft. Damit soll auf der einen Seite eine stringentere Ableitung gezielter Interventionen erleichtert werden, andererseits aber auch eine bessere Identifikation der zentralen Wirkfaktoren und Wirkprozesse psychologischer Therapien ermöglicht werden. Basierend auf einem europäischen Experten-Beratungsprozess wird ein EU-Programm 'The science of behavior change' angeregt.

\section{KARGER \\ Fax +497614520714

\section{(c) 2015 S. Karger GmbH, Freiburg}

$1016-6262 / 15 / 0252-0098 \$ 39.50 / 0$
Prof. Dr. Hans-Ulrich Wittchen 


\section{Core Role of Psychological Sciences for Psychotherapy, the Understanding of Mental Disorders and the Promotion of Mental Health}

Irrespective of the interdisciplinary nature of the mental health field, psychology is the 'mother' science for mental health and mental disorders and the essential science platform for both the development and the practice of interventions in the mental health field. Current shifts in research emphasis from traditional highly complex and dynamic psycho-bio-social behavioural interaction concepts to complementary putative causal neurobiological and broader biomedical research models have not changed the situation. In fact there is increasing convergence and synergy between biomedical and psychological research on mental health and mental disorders. Both fields, psychology and the biomedical disciplines, study the same or related phenomena with increasingly similar and complementary approaches and methods. Thus, it is not surprising that appraisals of strengths and weaknesses in both fields often arrive at the same conclusions [Schumann et al., 2014; Wittchen, 2014; Wittchen et al., 2014a]. Despite this continued trend of growing convergence and synergy, it seems to be timely not only to emphasize the unique contributions of the psychological sciences with regard to their traditions, theories, principles, and methods, but also to critically appraise their unique value, potential and impact on improving future research within the next few decades, for example with regard to a better understanding of the determinants and core mechanisms of mental health and mental disorders and the experimental and clinical derivations of improved interventions. Such an appraisal is of particular relevance for the field of psychotherapy or, more broadly, psychological interventions in general, as empirical evidence from clinical research suggests that cognitive-behavioural treatments (CBTs) are currently the most effective first-line treatments for many mental health problems and disorders [Butler, 2006; Emmelkamp et al., 2014].

However, the development of the psychotherapy field has been appraised as critical and some [Emmelkamp et al., 2014] have even suggested that psychotherapy research needs rebooting. For example, even after 25 years of research we still do not know why and how CBT works, or how to diminish the still-widening gap regarding the way psychological treatments are delivered in research settings as opposed to routine care. Under the still-considerable influence of historically rooted encapsulated schools of thought and increasing pressures due to the professionalization of psychotherapy, the field of psychotherapy seems to be confronted with the danger of losing its science basis and its essential roots in the psychological sciences. Latest progress in crucial basic, applied and clinical research in the psychological and neurobiological disciplines are frequently neither recognized nor translated into improved models or improved interventions and service delivery.

Because of this critical situation the EU has recently launched, supported and funded a major initiative called the 'Roadmap for Mental Health Research' [ROAMER, 2013; Haro et al., 2014]. As part of this project, a critical appraisal of the actual situation was conducted with the goal of providing guidance for an integrated and participatory roadmap for mental health research in Europe from the perspective of the Psychological Sciences. In this paper, we selectively summarize several core findings and conclusions of this programme (for a full discussion see the ROAMER supplement edition of the Int J Methods Psychiatr Res 2014;23,1-101).

\section{Relationship of Psychology and Psychotherapy}

Psychology can broadly be defined as an academic and applied discipline that involves the scientific study of (a) basic psychological functions such as perception, cognition, attention, emotion, motivation (b) complex psychological processes such as decision making, volition and behaviour control (c) their neural and biological underpinnings, as well as (d) personality, behaviour and interpersonal relationships. Psychology covers normal mental functions plus behaviours and addresses the questions of when, why and how they can become dysfunctional. Thus, psychology covers mental health and abnormal functions and behaviours, as found in mental disorders, with the goal of understanding individuals, groups and social systems. Psychology has been described as a hub science' [Cacioppo, 2007] with psychological findings linked to research and perspectives from the social sciences, natural sciences, medicine, and the humanities, such as philosophy. Over the past few centuries, the field of psychology has undergone several theoretical paradigm shifts (i.e. structuralism, functionalism, psychoanalysis, behaviourism, cognitivism) and is currently typically structured in subfields of which biological, experimental, developmental and clinical psychology have become closer to the biomedical field [Haslam and Lusher, 2011]. Depending on different national or regional scientific traditions, psychological and psychotherapy research can, however, also be grouped under social sciences, natural sciences or biomedical sciences, etc. In fact, in some countries terms like behavioural neuroscience or cognitive-affective neuroscience are used almost synonymously, leading to the field being denoted as psychological sciences.

Key corollaries of the psychological sciences are, e.g.:

- As compared to the biomedical field, the psychological sciences more explicitly emphasize a comprehensive interactional biopsycho-social approach to understanding and predicting a broad construct of 'behaviour' that refers to neurobiological, cognitive, affective and social-behavioural units of analyses and should not be misunderstood as denoting simply open motor behaviour.

- The relative role of biological, psychological and particularly social-environmental variables and their dynamic interplay in promoting normal and abnormal behaviour is examined within a 'dimensional' rather than a 'categorical' (diagnostic) approach.

- Consistent with a broad construct of behaviour, psychological research uses a variety of specific experimental and empirical methods (qualitative and quantitative, subjective and objective) and paradigms in human and animal research to observe causal and correlational relationships between psychosocial, environmental, psychological and biological variables. 
Psychological sciences emphasize environmental variables and a developmental perspective by appreciating the highly dynamic interplay over time, e.g. in psychological constructs of vulnerability - stress models and interactional constructs such as resilience and coping to understand behaviour change and its determinants.

\section{Defining Psychotherapy}

Based on such principles, constructs and methods, the science of psychology has also provided a set of unique methods and techniques for psychological interventions (i.e. psychotherapy) with the goal of preventing, treating and rehabilitating dysfunctional behaviour and mental disorders. Within this context of psychology, psychotherapy is defined as an applied clinical psychological domain discipline, dealing, generally spoken, with psychological treatments and interventions. More precisely psychotherapy has been defined as 'clinically relevant, empirically supported interventions of any type that are based on knowledge and expertise in the psychological sciences using psychological methods and means (as opposed to drugs as in psychiatry), typically by communication and/or behavioural exercises' [Wittchen and Hoyer, 2011, p 4].

This definition includes a large group of methods and approaches, developed to address the needs of patients and groups of patients with mental disorders or mental health problems, as well as their networks of support (e.g., partner and family) and covers prevention, treatment and rehabilitation in patients of all ages. Psychological treatments and interventions might range from highly sophisticated psychotherapy, delivered by specialized psychotherapists, to the application of specific behavioural techniques as part of a broader treatment plan (e.g., psychoeducation or motivational interviewing) by any health provider, including web-based and ehealth applications, whenever the criteria of the above definition are met and efficacy and/or effectiveness is established by randomized clinical trials or equivalent designs [van der Feltz-Cornelis and Adèr, 2000]. Because dysfunctional behaviour (also denoted in the literature as abnormal or clinically relevant behaviour or syndromes of mental disorders) has large and pervasive effects on health and ill health development and outcomes, there is a broad consensus in the scientific community that there is still vital to improve research with the goal to provide a better understanding of - the mechanisms underlying adaptive and dysfunctional behaviour,

- the developmental pathways and trajectories leading to dysfunctional and clinically significant behaviours as well as the determinants for these trajectories,

- the identification, by whatever means these determinants can be targeted and systematically influenced, and

- the mechanisms of behaviour change with regard to the promotion of normal, adaptive health as well as the reduction or prevention of dysfunctional and clinically significant behaviours as in mental disorders.

To reach these goals the ROAMER expert group [Schumann et al., 2014; Wittchen et al., 2014a] concluded that the currently frag- mented research field needs to be bundled under a broader comprehensive umbrella of a 'Science of Behaviour' programme in psychology and mental health research in general. For substantial progress to be made, the group suggested 3 essential steps: First, the adoption of an integrative conceptual framework; second, research to identify the most promising targets and procedures; and third, the improved translation of new findings and methods into a psychotherapy translational research agenda.

\section{Developing Conceptual Frameworks to Identify Aetiopathogenic Factors and Mechanisms to Derive Novel Therapeutic Targets}

There is consensus that improved knowledge about the core determinants of mental health and mental disorders is essential to identify the most promising therapeutic targets and procedures. There is also consensus that most mental disorders can be best characterized by their developmental and processual nature [Wittchen et al., 2014a,b]. To reach this goal, for internalizing disorders, examples of core questions that need to be answered include: Why are many people able to cope with traumatic stress and anxiety, while others develop increasingly severe avoidance behaviour resulting in anxiety disorders and their sequelae, such as helplessness and depression or substance use and eating disorders? What drives humans to develop and to maintain health risk behaviours such as harmful drinking and eating or smoking? Why are many individuals able to control their substance use or eating behaviours, while others develop a persistent maladaptive behavioural pattern ultimately leading to dependence or obesity, consequential disease and disability, and eventually a loss of control over their life? And, more generally: What are the critical trajectories from maladaptive behaviours to (mental) disorders as defined in classificatory diagnostic systems, such as the Diagnostic and Statistical Manual of Mental Disorders (DSM) [APA, 2000, 2013] or the International Classification of Diseases [WHO, 1991]? Can we modify critical developmental pathways by directly targeting such mechanisms?

From a psychological and cognitive-affective neuroscience perspective, such health-risk behaviours and mental disorders can be conceptualized as the procedural and developmental outcome of complex interactions of individual genetic predispositions with the environment and as neurobiological, psychological and social processes within 'personal biographies'. The brain as the essential target structure is shaped by genetic factors, development, environment and experience in multiple, highly complex and probably individual ways. All human actions including adaptive and dysfunctional decision making involved in health-risk behaviour and common mental disorders are the result of complex interactions between: (1) higher cognitive processes such as the anticipation of long-term consequences, the regulation of emotional impulses and the inhibition of habitual responses in favour of long-term goals; (2) complementary forms of learning and memory, and (3) basic emotional, reward-related, and motivational processes. Health-risk 
behaviours and mental disorders also provide indirect evidence for latent shared mechanisms. These communal behavioural, cognitive and physiological features suggest the existence of similar aetiological mechanisms, such as the 'continued making of maladaptive choices even in the face of the desire to make a different choice' as a defining diagnostic criterion [APA, 2000]. They frequently 'bundle together', start predominantly early in life and escalate temporally in similar patterns. In this context, mental disorders can be conceptualized as yet insufficiently understood dysfunctions in basic psychological processes as well as 'perturbations' in brain functions at the cell and systems level. The former processes can be conceived as being centrally involved in the behavioural, cognitiveaffective and somatic symptoms currently used to define mental disorders. The latter 'perturbations' can broadly be described as various types of dysfunctions in complex structural and functional neural circuits for information processing. Appropriate experimental psychological paradigms in combination with brain imaging methods, supplemented by molecular and biochemical analyses, allow examining the complex, plastic interactions and the connectivity of brain regions for different functions.

At present we still lack complete maps of such human circuitry dysfunctions. For many conditions, however, candidate models exist and serve as a starting point (e.g. addictive behaviours - involvement of reward circuitries [Koob and Le Moal, 2001]; fear and anxiety disorders - abnormalities of the amygdala-based fear circuitry [Shin and Liberzon, 2010]). Corresponding candidate mechanisms at the behavioural level include aspects of cognitive control and volition, emotion regulation, meta-cognition, and decision-related processes, such as evaluation, risk perception and temporal discounting. However, the diagnostic specificity, moderating and even causal role of such highly complex 'circuitry perturbations' in the evolution of mental disorders, persistent health-risk behaviours and associated clinical behavioural syndromes (e.g. adipositas, obesity) remain unclear. Despite impressive achievements in this area, we still lack answers to core questions [Goschke, 2014].

How do complementary cognitive, affective, and motivational systems interact dynamically, which proximal and distal variables (e.g. acute or chronic stress) modulate patterns of interactions among these systems, and how do stable patterns of system interactions evolve as a result of genetic variation, developmental change, and learning?

Which mechanisms underlie transitions from adaptive cognitive-behavioural patterns into maladaptive patterns of persistent health-risk behaviours or mental disorders? Are there critical developmental windows of vulnerability? Are cognitive dysfunctions (e.g. impaired volitional control) simply 'constitutive markers' or generalizable causal disease factors? How can effective change of dysfunctional behaviour patterns be induced and maintained by targeting such mechanisms?

One promising way to address such questions is to conceptualize mental disorders as maladaptive developmental processes, in that they reflect complex interactions of individual genetic predispositions with environments within 'personal biographies' of psychological dispositions, associated with characteristic changes in neural systems and circuitries. One might also hypothesize that processes responsible for the development of health-risk behaviours and the shaping of respective vulnerabilities can be conceptualized on the same theoretical grounds and that both unique and cross-cutting pathways and mechanisms exist. Such an approach offers the opportunity of addressing core questions; however, such an endeavour involves addressing a number of critical issues considered below.

Cognitive-affective factors and action: There is a need for concerted efforts to examine experimentally the neurocognitive mechanisms underlying human behaviour and, for example, decision making. This requires a better knowledge of cognitive systems [Schumann et al., 2014], including modulating influences (i.e. stress [Schumann et al., 2014]) and developmental (see below) trajectories of adaptive and maladaptive patterns, such as of decision making and action control, in non-clinical and clinical populations [Goschke, 2013].

Developmental mechanisms and pathways: Mental disorders and health-risk behaviours must be conceptualized as occurring on multiple time scales and thus as 'developmental' in a broad sense. The concerted growth of regulatory mechanisms in early ages is a prerequisite for personal autonomy, adaptive behaviour and sustained mental health. The brain's developmental plasticity in early ages along with prospective longitudinal evidence that the onset of most common mental disorders is centred in relatively small, predominantly early-time windows in the lifespan [Kessler et al., 2005; Beesdo-Baum and Knappe, 2012] renders a developmental psychological perspective essential. Further, determinants and modifiers of (mal-)adaptation might arise from genetic/epigenetic factors as well as from gene-environment interactions, all intersecting at the level of functional and structural neurobiological functioning during development, progressing from early pre- and postnatal stages throughout the lifespan.

Stress and environment: There is overwhelming evidence that conditions of traumatic and chronic stress can significantly change the structure and functioning of brain circuits and significantly impact gene expression and translation. Such plastic changes in peripheral physiology and neural activity are found to be linked to adverse health outcome, as characteristic differences in neurotransmitter systems, immune systems functions, and peripheral endocrine patterns follow stress exposition. Thus, acute and chronic stress affect the developmental trajectory of health-risk behaviours in humans and animals. Use of cutting-edge psychoneuroendocrine methods are needed to understand the complex interaction patterns of stress and ill-health behaviour in children, adolescents, and adults. Innovative methods to measure 'stress' will propel our understanding of the mechanisms leading to health-risk behaviour and resulting morbidity.

Computational and other modelling approaches (e.g. trajectory, latent growth modelling): These are central for modelling complex interactions to reduce the enormous complexity because there are no simple 'causal' relationships. There is a lack of explicit (e.g. computational or connectionist) models of the mechanisms and dynamics of developmental change, which are needed for under- 
standing how nonlinear interactions among component processes on different levels of analysis give rise to emergent properties (e.g. nonlinear developmental trajectories, critical periods, stable patterns of dysfunctional regulation).

Associated 'reductionistic' mechanistic basic research: Such research is needed ultimately to identify and examine the nature of the characteristics of critical systems and putative causal mechanisms. This means that such an overall strategy needs to be supplemented by stringent behavioural and biological studies. This applies to interactions of putative components within neural cells, cell systems and circuitries, within different psychological processes and between neural circuitry and psychological processes. Skilful data integration and modelling (systems biology, computational neuroscience) can help to reduce complexity so that specific hypotheses become testable in concrete biological or behavioural models, which in turn can feed back into the research design [e.g., Kraemer, 2014; Siegle et al., 2012].

Epidemiology and population genetics: Clinical samples have only limited value for investigating potentially causal interactions in many conditions. This is not only due to the fact that subjects sampled from clinical population are typically suffering for many years, but also due to confounding by selection bias, treatment effects and the presence of comorbid conditions that might have been developed as a consequence of prior clinical conditions. They also provide little information about normal and adaptive processes, protective factors or preventive targets, limiting their value when it comes to identifying protective factors and resilience. Use of the full range of options provided by descriptive and causal analytic epidemiological approaches to define cases/patients in all stages, as well as population genetics and developmental psychology to define developmentally sensitive, prospective cohorts of relevant pheno- and genotypes are thus mandatory to identify developmental and symptom pathways and their critical trajectories.

Clinical, treatment and translational perspective: Identifying the potentially causal or mediating role of such processes and interactions over time promises a better understanding of when, how and why the evolved mechanisms might fail or become dysfunctional. Such insight can be seen as a precondition for testing similarities and differences in the evolution of specific behaviours and syndromes and their malleability. More importantly, such improved understanding also provides the opportunity to identify potentially more promising targets of intervention that aim less on modifying common symptoms, but rather directly allow the underlying core vulnerabilities and core dysfunctional aetiological factors to be modified. This might have tremendous potential, e.g. for optimizing targets and form and dose of cognitive-behavioural intervention in patients as well as preventive efforts.

\section{Symptom Progression Models and an Integrative Translational} Public Health Perspective

From an integrative translational perspective existing 'symptom progression models' are at least of great heuristic value (fig. 1). The existing descriptive models, although imperfect and still largely speculative, assume a systematic evolution of symptoms from ini-

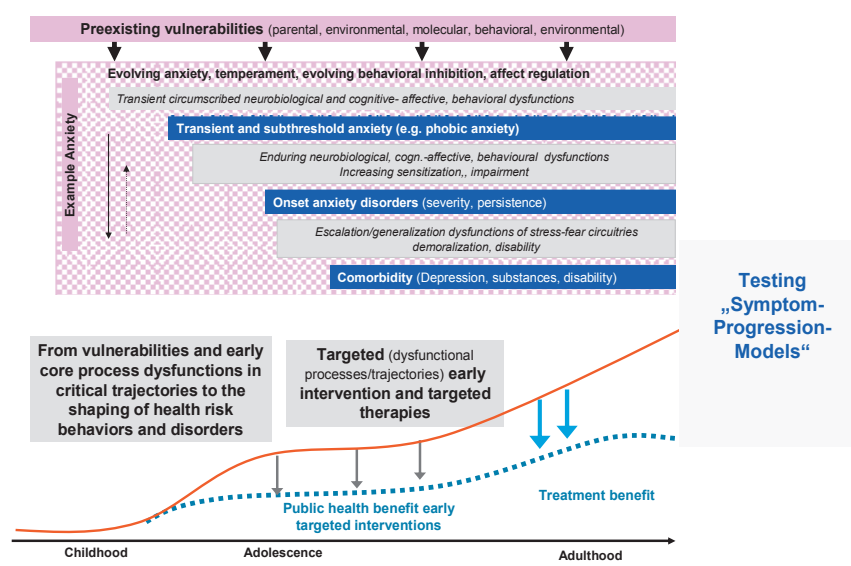

Fig. 1. Examples for symptom progression models.

tially transient to more persisting and pronounced expressions, seen as the result of critical interactions of vulnerabilities with environmental and experiential influences and their dysfunctional processing in developmentally sensitive periods.

\section{Identifying the Most Promising Targets}

Empirically sound models are instrumental for defining starting points, for example with regard to questions like: 'What are putative core dysfunctional process targets? What are the most critical 'trajectories' in the developmental pattern? What is a promising and feasible strategy to modify the progression trajectories form early preclinical signs to clinically significant expressions. Such a targeted strategy not only has the potential of improving existing and deriving novel interventions that could be adapted to early preclinical and advanced clinical stages, but also has two evident additional advances. First, such an approach is likely to enhance the public health utility of research, because there is the premise that such early targeted intervention will be associated with a higher probability of substantially reducing the future burden. Second, this strategy will at least partially avoid the traditional 'translational hurdles' of the conventional approach (fig. 2).

Procedural developmental modelling in representative samples of both low- and high-risk subjects and of clinical groups is required to identify: (1) the determinants of critical trajectories (e.g. adaptive to maladaptive, non-clinical to clinical), (2) specific and cross-cutting processes, and (3) novel 'targeted' interventions. It further carries the potential for greater research synergies across disciplinary boundaries, and adds considerable public health and translational utility, beyond major impact on diagnosis and treatment.

As displayed in figure 2, such a strategy requires an iterative transdisciplinary multi-method approach in samples and cohorts starting with many questions as early as conception or (very) young age up to old age in order to

- elucidate the 'normative' (adaptive) expressions of psychological functions and processes, their neurobiological, molecular and genetic substrates, and their interaction by developmental 
stage in well-defined 'normal' populations with theory-driven, tailored task paradigms;

- model pathways and system interactions both at a structural level (e.g. latent trait and growth, reduction of complexity, 'higher order constructs') and a 'mechanistic' level (e.g. circuitry processes, computational neuroscience);

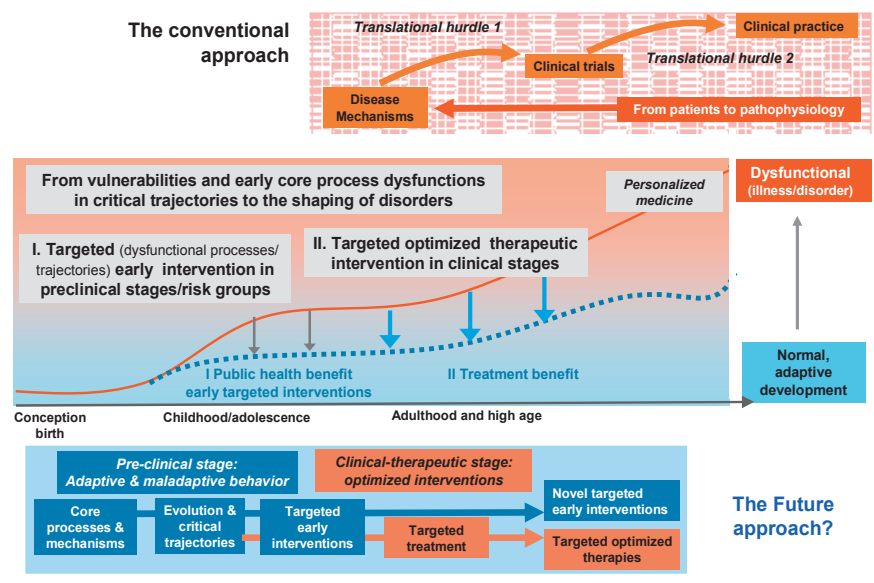

Fig. 2. The conceptual framework.
- validate, challenge and improve the emerging models by basic science experiments (e.g. cytological studies) to derive improved models for further testing (e.g. systems biology);

- incorporate individual and contextual factors to determine how functions, processes and circuitries are developmentally influenced by learning, plasticity, emotional traits, and stress;

- apply the derived models in defined risk cohorts to examine how, why, when and where system components are affected and prospectively associated with increased risk for onset; and

- test, ultimately, how and to which degree these dysfunctions can be altered by interventions that specifically target the underlying mechanisms.

We recently - together with many colleagues - elaborated tentatively matching research strategies for such an agenda using 4 specific examples of syndromes and disorders, namely 'stress, fear, avoidance and anxiety disorders', 'addictive behaviours', 'impulse control disorders', and 'eating and metabolic disorder' [Wittchen et al., 2014a]. Some of the core goals and core challenges involved in such a programmatic process are depicted in table 1.

These examples make it clear that in order to make major progress in mental health research and mental disorders we have to redirect our current fragmented disciplinary research strategies to-
Table 1. Goals and challenges for selected behavioural problems and related mental disorders

\begin{tabular}{|c|c|}
\hline Goals & Challenges (advances needed) \\
\hline $\begin{array}{l}\text { Analysis of the development of } \\
\text { adaptive and maladaptive } \\
\text { mechanisms and modulators of } \\
\text { decision making and behavioural } \\
\text { control }\end{array}$ & $\begin{array}{l}\text { How habitual, motivational, affective, and executive control systems interact dynamically and } \\
\text { how this interaction is modulated by proximal and distal variables (e.g. acute/chronic stress); } \\
\text { how stable patterns of interactions between motivational, emotional and executive-control } \\
\text { systems evolve as a result of the interplay of learning, development, and genetic variation, and } \\
\text { how inter-individual differences in intra-individual change patterns emerge; } \\
\text { which mechanisms underlie the transition from adaptive goal-directed behaviour into } \\
\text { dysfunctional regulation patterns leading to maladaptive behavioural choice; } \\
\text { whether behavioural change and adaptive (e.g. health-promoting) behaviour can best be } \\
\text { supported by strengthening volitional functions (e.g. the ability to maintain intentions in the } \\
\text { face of competing habits or emotional temptations) or by enhancing motivational incentives } \\
\text { associated with long-term goals (or whether both aspects need to be targeted). }\end{array}$ \\
\hline $\begin{array}{l}\text { Addictive behaviours and } \\
\text { addiction }\end{array}$ & $\begin{array}{l}\text { Strategically intermediate and long-term objectives might build on novel theoretical unified } \\
\text { perspectives for addiction as 'vulnerabilities' in established 'decision-making systems' [Redish } \\
\text { et al., 2008] and extensions thereof (e.g. temporal difference reinforcement learning). }\end{array}$ \\
\hline $\begin{array}{l}\text { Eating, eating disorders and } \\
\text { neurometabolic conditions }\end{array}$ & $\begin{array}{l}\text { Examination of common and specific psychological, neurological and circuitry dysfunctions } \\
\text { across various forms of syndromes and disorders and in different stages of expression/develop- } \\
\text { mental stage may help to derive improved models and novel therapeutic targets and approaches; } \\
\text { although multiple candidate mechanisms have been proposed with symptom and diagnostic } \\
\text { relevance for Anorexia Nervosa, evidence for other eating disorders and related phenomena are } \\
\text { lacking; } \\
\text { Determination of whether leptin resistance develops in the homeostatic circuitry controlling } \\
\text { appetite and, thereby, a similar resistance develops in the reward circuitry. If so, food may serve } \\
\text { as a rewarding stimulus, independently of energy balance, and alteration of these mechanisms } \\
\text { may facilitate understanding of obesity. }\end{array}$ \\
\hline $\begin{array}{l}\text { Stress, fear, avoidance and } \\
\text { anxiety disorders }\end{array}$ & $\begin{array}{l}\text { Despite the existence of various heuristic models, it is not known why, when and how which } \\
\text { components of cognitive-behavioural treatment (CBT) are essential in promoting behaviour } \\
\text { change. Neurobiological and particular fear-circuitry underpinnings of this method remain } \\
\text { unclear. Improved understanding of such processes might have tremendous implications not } \\
\text { only in improving treatment by optimizing the formal and content structure of CBT, but also } \\
\text { for targeted prevention of anxiety-related reactions and behaviours, before the full syndrome } \\
\text { is expressed clinically. }\end{array}$ \\
\hline
\end{tabular}


wards a broader behavioural science focus. Most solutions to the complex problems of mental health require the synthesis of knowledge and methods across various disciplines within a psychological framework. However, past barriers have made true interdisciplinary approaches rare exceptions [Pellmar and Eisenberg, 2000]. The fragmentation and 'disciplinary insularity' of the mental health research field needs to be overcome by linkages among relevant biological, psychological, social and clinical approaches. Thus, only a concerted trans-disciplinary effort will be able to adequately address the full scope of mental health, involving behavioural, clinical and neuroscience concepts using a multi-level, multi-measurement approach within a developmental framework. Our proposal refers in this context to similar conceptual frameworks, such as the one proposed by the NIMH Working Group on Child and Adolescent Mental Health [NAMHC, 2008] highlighting the critical developmental transition points across the lifespan in order to link basic science findings with clinical and social aspects.

\section{Advancing Psychotherapy Research}

Against the background of the previous considerations regarding psychological theories and models of mental health and disorders, the following question arises: How can the painted perspective be brought into improved psychotherapy research? Given the current quite diffuse situation in research and practice of psychotherapy, one might also ask whether the time has come to reboot psychotherapy research altogether. Psychotherapy is rooted in many different traditions, conceptual models and 'schools of thinking', ranging from theoretically based approaches, such as psychodynamic therapy, to more experimentally based psychological interventions, such as CBT. Psychotherapy, although having some well-founded specific theories and models, research targets, methods and procedures, in many respects does not have an overarching scientific foundation. Clear conceptual frameworks that bring together the wide variety of findings, models and perspectives and essential linkages to basic psychological and neurobiological research evidence are lacking. Further, attempts to develop common overarching integrative frameworks, such as proposals for empirically based 'psychological therapies' or a 'unified treatment' have not been successful so far. Thus, it is not surprising that there is a tremendous gap between psychotherapy methods as applied in well-controlled clinical trials and psychotherapy in routine care. In particular, it is disturbing that it is not clear what is meant when mental health interventions in real life are described as psychotherapy or even CBT, because frequently neither the content, nor the form and structure of routine care psychotherapy matches to that done in well-controlled clinical studies. Nevertheless, in policy making we use the efficacy and effectiveness results of such trials as justification for the claim that psychotherapy works. This incoherent picture of psychotherapy has created increasing confusion for policy makers and the public with regard to the scientific status of psychotherapy and the misconception that psychotherapy is little more than counselling.

\section{A Way forward to Reboot Psychotherapy Research?}

Given the established efficacy and benefits of psychotherapy and CBT in clinical controlled trials, the time has come for concerted action to develop psychological interventions and psychotherapy further on the grounds of the psychological sciences. Without trying to be complete and without implying that current established psychotherapy research areas should be given up, we want to give a few examples how we can make major progress in this respect.

\section{Focus on Principles of Behaviour Change Targeting Core}

Processes

In the preceding parts of this paper, we have emphasized the general strategies that seem to be timely and most promising, i.e. to focus on experimentally and empirically supported principles of behaviour change, and targeting the relevant core processes involved in the aetiology and maintenance of mental disorders and mental health problems. Mental disorders can be regarded as 'functional and developmental' in nature, as has been outlined above. Formally, we can conceptualize mental disorders in terms of (disturbances in) core psychological functions (attention, learning, memory, decision making, cognitive control, emotion regulation, etc.; see [Goschke, 2014]) or in terms of their meaning and function (e.g. a bias towards over-general autobiographical memory retrieval in depression, although detrimental for everyday problem solving, may help ward off unpleasant, traumatic or threatening memories; [Williams et al., 2007]). Although this innovative approach, mostly using interventions relying on ideas gleaned from experimental cognitive psychology, is still in its early developmental phase, it nevertheless has already provided some remarkable findings in clinical samples (e.g. memory specificity training for depression, see [Raes et al., 2009; Neshat-Doost et al., 2013]; executive function training for attention deficit hyperactivity disorder (ADHD), e.g. [Van der Oord et al., in press]; attentional bias modification training for alcohol abuse, e.g. [Schoenmakers et al., 2010]; motivational interviewing techniques in behaviour therapy interventions, e.g. [Geller and Dunn, 2011]). Other examples include interventions that use knowledge and principles gleaned from inhibitory learning and inhibitory regulation models in anxiety in order to optimize extinction learning [Craske, 2015].

\section{Combining Traditional Diagnostic with Relevant}

Endophenotypes Approaches

It has become evident that existing diagnostic classification systems have notorious weaknesses that may be an obstacle for the development of improved alternative diagnostic approaches [Fava et al., 2014]. Targeting core psychological dysfunctions and processes rather than sticking to traditional psychopathological syndromes and the criteria of specific mental disorder (as defined in DSM-5) is seen as a promising additional strategy, closely linked to the endophenotype approach that has become a main topic in neurobiological research. Endophenotypes or intermediate phenotypes are specific clusters of symptoms that constitute building blocks or 
intermediate processes for mental disorders and supposedly carry a more specific and consistent genetic load than the overall disorders that they contribute to [Skuse, 2001]. In the common diathesisstress models of psychopathology and mental disorders, endophenotypes arguably represent vulnerability factors or (bio-)markers for mental disorders rather than being indicators for the full-blown disorder. Such endophenotypes can often be related to specific functions in a cognitive psychology framework, such as memory, attention, executive functions, or others. In some domains, considerable progress has already been made in the identification of meaningful cognitive endophenotypes over the past few decades. For instance, impulsivity as measured by a number of tasks and instruments is not only a promising core endophenotype for ADHD, but also for substance disorders and other externalizing disorders. Dysfunction in emotion dysregulation is involved in a variety of mood and anxiety disorders [Hofmann et al., 2012]. Autobiographical memory biases may be involved in obsessive-compulsive disorders, trauma and depression [Williams et al., 2007]. Executive function deficits including impaired working memory and inhibition and increased impulsivity are endophenotypic not only of ADHD but also of addiction, obsessive-compulsive disorders and eating disorders [Robbins et al., 2011]. Repetitive negative thinking is a cognitive marker of mental disorders as diverse as depression, anxiety disorders, bipolar disorder and psychosis [Ehring and Watkins, 2008]. It can be expected that future interventions will increasingly consist of evidence-based treatment programmes targeting specific psychological processes (e.g. memory specificity training for mood disorders and post-traumatic stress disorder (PTSD), executive function training in $\mathrm{ADHD}$, addiction and eating disorders, attentional bias modification training for addiction and anxiety disorders) in place of school-of-thought-based or disorder-based interventions. Findings from such approaches might fit much better with a modular psychology framework and a dimensional endophenotype perspective than with a categorical, nomothetic view of mental disorders. However, it should be emphasized that the search for improved endophenotypes and matching interventions should not be misunderstood as a departure from traditional psychopathology and traditional diagnostic concepts. Traditional diagnostic classification systems such as DSM-IV and DSM-5 will remain in the future - despite inherent weaknesses, representing the essential link between science and research on one side and clinical practice and the management of health care systems on the other.

\section{Focus on Understanding Why Psychotherapy is Efficacious and Effective}

There is little doubt from a scientific perspective that CBTs and some other psychological interventions are effective, highly beneficial and cost effective for a wide range of mental disorders and health conditions (see e.g. Health Technology Assessment reviews; [Roth and Fonagy, 1996; Butler et al., 2006]), such as anxiety, stress and trauma-related disorders, depressive and somatoform and pain disorders, personality disorders, substance use disorders and behavioural addictions, eating disorders and a number of childhood disorders [Hofmann and Smits, 2008]. For all these disorders, various variants of CBT have been established in clinical randomized trials. There is also strong evidence for the efficacy of CBTs and other psychotherapeutic methods, at least as an adjuvant treatment, for psychotic and bipolar disorder [Jones et al., 2012a,b; van der Gaag et al., 2012; Hutton and Taylor, 2013]. Further, psychotherapy is established for a wide range of somatic diseases and a range of health conditions for which behavioural factors play a significant role in the initiation, maintenance and rehabilitation, such as cancer (e.g. [Fors et al., 2011]), cardiovascular disease (e.g. [Gulliksson et al., 2011]), metabolic disease and obesity (e.g. [Grilo et al., 2012]), and fibromyalgia [Kashikar-Zuck et al., 2012].

In addition, there are multiple systems regularly evaluating evidence-based psychological therapy, such as the National Institute for Health and Clinical Excellence (NICE) Guidelines (www.nice. org.uk), the APA/Division 12/Society for Science of Clinical Psychology/SSCP platform or Cochrane reviews (www.cochrane.org). Despite this overwhelming level of evidence, there are, however, some notable problems that should be resolved in future research.

\section{Identifying Core Ingredients and Mechanisms of Action}

Current systems of evaluating evidence-based psychotherapy focus on data supporting (psycho)therapeutic packages, while neglecting the degree of empirical support for the theoretical basis of these packages. To delineate core ingredients and to identify mechanisms of action and therapeutic change, David and Montgomery [2011] proposed a new grid system for the evaluation of evidencebased psychotherapy, proposing 9 categories, resulting from levels of evidence regarding 2 factors: (1) theory/mechanisms of change and (2) interventions (i.e. therapeutic package) derived from that theory. The proposed categories are not static, but based on the progress of research, allowing one form of psychotherapy to move from one category to another. On the level of the theoretical and mechanisms of change, it should be emphasized that this level does not necessarily refer to the aetiopathogenic mechanisms that have led to expression of the disorder or condition, but more generally to the assumed mechanism of change in the psychotherapeutic approach under study. Mediation and moderation analyses are typically involved in this kind of analysis. The hypothesized mediators and/or moderators should be specified in advance, based on scientific clinical theories. The specific role of 'placebo conditions' [Kirsch, 1990] and non-specific 'contextual' factors, such as therapeutic alliance [Wampold, 2001], in psychotherapy should be emphasized. On the outcome level, analyses focus on the impact that a specific intervention has on a specific outcome or on a spectrum of outcomes (e.g. clinical conditions). The quality of the outcome analysis depends on the level of outcome specification, i.e.: (1) the clinical symptomatology (i.e. including primary and secondary symptom or diagnostic outcomes); (2) the general level of distress; and (3) the social functioning and quality of life to understand how the clinical condition(s) and/or interventions impact the real life of our clients. Cost-effectiveness considerations should be incorpo- 
rated as a standard, because even the best treatment with known mechanisms of action is unlikely to be used if it is too expensive [Sava et al., 2009].

Despite its claim as being scientifically based, current CBT approaches and manuals are not entirely consistent with novel basic and clinical research (i.e. neural underpinnings, fear circuitries, (epi-)genetic factors, developmental pathways, dynamic role of learning, extinction, avoidance, safety behaviour etc., [Etkin and Wager, 2007; Andrews et al., 2009; Arch and Craske, 2009; Gloster et al., 2011; Kircher et al., 2012]). Emotional responses like fear and anxiety are grounded in 'complex systems' that feed back to sensory systems, heightening vigilance and information gathering (if a threat is expected or detected) and, importantly, prompting for example defensive reflexive, autonomic, and motor responses that in evolutionary history acted directly to counter threats and escape punishments [Arch and Craske, 2009]. Comparative research across different forms of mental disorders is necessary to detect such core aspects of dysfunctions shared by all disorders in contrast to disorder-specific features [Etkin and Wager, 2007; Haber and Rauch, 2010; Lueken et al., 2011; Domschke and Deckert, 2012] and to inform how therapeutic interventions can be optimized, e.g. by component, mediator and moderator analyses. A better understanding of the basic mechanisms of mental disorders and their personal variations will guide the translational agenda of how evidence-based psychotherapy can be procedurally optimized, e.g. for more effective reduction of dysfunctional patterns of fear, anxiety and avoidance. Such research will also provide information on when best to intervene with which component (i.e. timing of interventions, add-on modules, and comorbidity issues). Here, there is a clear need of research in truly clinical samples or even clinical cohort samples, rather than in (psychology) students with heightened scores on a questionnaire.

More rigorous designs are needed to investigate mediators of treatment outcome. Current findings are inconclusive. Little is known about the similarities, differences and specificity of the change processes across treatment modalities and paradigms. There is a clear need to examine how these treatments work and what accounts for the variability in outcomes. Unfortunately, few studies have measured the proposed mechanisms during treatment, so it is unclear whether change in the supposed mediator precedes change in the outcome. For example, although most therapists hold that change in cognitions is responsible for effects achieved with CBTs, there is still a surprisingly small number of studies that have directly tested for cognitive mediation (e.g. [Meyerbröker et al., 2013]). Most of the studies that focused on mediators of treatment outcome failed to assess whehter the mediator changed before the improvement occurred, meaning it was not possible to test questions of temporality, a critical component of mediation [Teachman, 2014].

Focus on Understanding and Tailoring Combined Treatments

A controversial question is still the combination of drug and psychological treatment. Whereas there are typically beneficial additional effects when psychotherapy is added to drug therapy, the reverse does not necessarily seem to be the case. A number of studies have investigated the combination of psychotherapy with antidepressant drugs or tranquilizers, but generally such pharmacotherapy did not enhance the effects of evidence-based psychotherapy as stand-alone treatment (e.g. [Forand et al., 2013]). Given that many patients asking for psychotherapy have some form of past and/or concurrent medication and given the unfavourable cost-effectiveness ratio of combined treatment approaches, there is an urgent need to answer this unresolved issue and to investigate the moderator role of concurrent therapies. There is little systematic research about how such medication affects psychotherapy and what clinicians and researchers should do in such situations. Unresolved core questions are: (1) Do past or only concomitant medication affect treatment response and the mechanism of action? What type and pattern of medication has an impact? (2) How frequent are particular patient constellations: Is the patient a non- or partial responder associated with appropriate or inappropriate psychopharmacotherapy? Would the patient profit more from stopping or changing (optimizing) medication? (for further questions see table 2). Currently, tapering off medication before inclusion or keeping the medication constant (combination treatment) are the 2 major options, providing no further or more specific guidance. Integration of psychological and pharmacological interventions may bear great promise for the treatment of some aspects of psychopathology (particularly disordered memory processes); however, much more knowledge is needed on the exact boundary conditions for pharmacological intervention on specific psychological processes. Increased knowledge along those lines should help to pave the way for interventions that combine psychological and biomedical techniques in an intelligent, science-based way and/or follow a clinically meaningful sequential strategy [Fava, 2000]. Furthermore, the development of empirically grounded strategies for switching between and within pharmacological and psychotherapeutic treatments in case of non- or insufficient response is an important challenge for the future (e.g. [Gloster et al., 2015]).

\section{Exploring the Potential of Novel Technologies}

Psychotherapy might profit in research and practice from a systematic exploration of e-health technologies. Beyond the option of providing improved and ecologically valid information about the patients' behaviour in real life, they might allow optimizing essential components of the treatment outside the therapy setting. To make a better contribution to reducing the disease burden of mental disorders, psychotherapy research might also systematically explore internet-based treatments and e-Mental health tools (e.g. to support 'real time' clinical decision making to prevent treatment failure or relapse). In this field, 4 broad research areas can be distinguished (for a broader discussion see [Emmelkamp et al., 2014]): (1) research on how to apply the fact, in routine mental health care, that internetbased treatments are efficacious; (2) further development of existing internet-based treatments; (3) the use of technological innovations, such as a move to the mobile phone, 'serious gaming' [Merry et al., 2012], and the use of avatars; and (4) generic issues of dosage and feedback with the aim of reducing treatment failures. 
Table 2. Goals and needs for future research in psychological science

\begin{tabular}{|c|c|}
\hline Goals & Challenges (advances needed) \\
\hline $\begin{array}{l}\text { Improved basic research aiming } \\
\text { towards a better understanding } \\
\text { of the basic mechanisms of } \\
\text { behaviour initiation and } \\
\text { maintenance }\end{array}$ & $\begin{array}{l}\text { Analyses of basic psychological functions (i.e. attention) and processes (i.e. learning) and - } \\
\text { even more pronounced - of cognitive factors of higher order (decision making, memory, } \\
\text { impulsivity, motivation, etc.), including their neurobiological underpinnings. }\end{array}$ \\
\hline $\begin{array}{l}\text { Examining how psychological } \\
\text { factors affect health-related } \\
\text { behaviour }\end{array}$ & $\begin{array}{l}\text { Research on how such psychological factors and mechanisms influence health-related } \\
\text { behaviours, their initiation, maintenance and 'spontaneous natural' change, particularly with } \\
\text { regard to determinants for their critical trajectories. Such research is seen as high priority for } \\
\text { an improved basic understanding of mental health and mental disorders and normal versus } \\
\text { dysfunctional behaviour. }\end{array}$ \\
\hline Development over the lifespan & $\begin{array}{l}\text { Research that examines such domains over the lifespan and specifies how vulnerabilities and } \\
\text { stress influence critical developmental trajectories to poor health and specific mental disorders } \\
\text { in particular. There is a strong need for improved developmentally sensitive models of mental } \\
\text { health over the whole lifespan but in particular in childhood and adolescence as the core } \\
\text { high-risk period for onset of mental disorders. }\end{array}$ \\
\hline $\begin{array}{l}\text { Moderators and mediators } \\
\text { involved in behaviour change } \\
\text { (treatment) }\end{array}$ & $\begin{array}{l}\text { Manifest research gaps exist with regard to the mechanisms, moderators and mediators in- } \\
\text { volved in behaviour change within the specific context of psychological interventions and in } \\
\text { particular CBT. This involves core questions such as: What are the neural and neurobiological } \\
\text { changes associated with CBT-induced change? What are active ingredients and mechanisms } \\
\text { of effective and efficacious psychotherapy? }\end{array}$ \\
\hline $\begin{array}{l}\text { Improved translation of basic } \\
\text { and applied psychological } \\
\text { research into application and } \\
\text { practice }\end{array}$ & $\begin{array}{l}\text { There is a considerable science-practice gap on various core levels of research } \\
\text { - Improved aetiopathogenetic research that systematically incorporates social-environmental } \\
\text { context and determinants as well as the neural underpinnings, based on models of mental } \\
\text { health development and mental disorders. } \\
\text { - Improved diagnostic assessment procedures targeting the core aetiopathogenic processes, } \\
\text { rather than simply targeting psychopathological features as specified in existing, although } \\
\text { imperfect, diagnostic classificatory rules. } \\
\text { - Improved clinical intervention research to gain a better understanding of CBTs by } \\
\text { identifying the core active ingredients, core mechanisms. } \\
\text { - «Improved targeted intervention modules for preventive and treatment purposes } \\
\text { - Improved models and approaches of dissemination and translation to routine care. }\end{array}$ \\
\hline
\end{tabular}

\section{Reducing Translational Barriers}

Although effective psychological treatments (CBTs) exist for many disorders, treatment rates are low [Wittchen et al., 2011] and are characterized by long time lapses (typically decades) between disorder onset and treatment [Wang et al., 2005; Gustavsson et al., 2011; Wittchen et al., 2011]. Among the few patients treated, drug and particularly psychotherapy treatment are frequently inadequate or inappropriate. State of the art 'CBTs' as studied in randomized controlled clinical trials (RCTs) are rarely applied, despite short- and long-term effect sizes that are larger than for other treatments of mental disorders. When applied, treatment characteristics in routine care typically do not match the RCT manual specification or treatment guidelines in terms of type, length and content. The reasons for this unfortunate situation are only partly understood despite promising suggestions for how to change the situation by use of improved science-practice networks [Crawcour et al., 2012]. Increasing the 'ecological validity' and clinical utility of CBT programs by avoiding overly selective inclusion criteria and diagnostic fragmentation might provide guidance on how to deal with comorbid conditions and prior or concomitant drug treatment and with patients not achieving full remission.

\section{Conclusion: Goals and Needs in Psychological and Psychotherapy Research}

Great progress has been made over the past decade with regard to the identification of a range of options for how to develop an improved understanding of mental disorders and their aetiopathogenic pathways across the lifespan. At the same time, firmer evidence has become available that CBTs and some other psychological treatments are effective in most mental disorders and mental health problems. However, we still have only rudimentary knowledge about the question of why people develop mental disorders. Our effective methods are far from being satisfactory and their translation into the community to reduce the burden of mental disorders overall is largely deficient.

Given the established efficacy and benefits of psychotherapy and CBTs in particular, time has now come for concerted action to overcome the current fragmentation of research areas and fields. It is time to launch a comprehensive research agenda with clinical trials, and basic and translational projects within an overall populationbased developmental framework covering all ages, instead of a smaller-scale, narrow research agenda. We have outlined a number 
Table 3. Gaps and needs in research on e-Mental health and innovation of mental health care [Emmelkamp et al., 2014]
Gaps and needs in research on e-Mental health and innovation of mental health care

It is not clear when and how each of the treatment formats of psychological treatments (individual; group; family; guided self-help; Internet-based) is able to help better.

A shift in outcome research is needed towards more focus on reaching the right target groups, realizing effects in populations instead of individuals, reductions in disease burden and quality of life and improvement of coverage of treatments.

For individual therapy generic issues of dosage and feedback with the aim of reducing treatment failures in the context of e-mental health should be further investigated.

A portfolio of models to deliver psychological treatments should be developed along with methods to examine these models from a public health point of view.

More research on applying and implementing e-mental health tools and Internet-based treatment for mental disorders in several specific fields is needed

Prevention of mental disorders and public health.

Treatment of mental disorders in primary care.

Treatment of mental disorders in specialized mental health care.

General medical settings.

New and better Internet-based treatments should be developed

Comparative research on different treatment formats and unguided self-help can help in answering which treatment format helps best in which context.

More research is needed on how and in which setting Internet-based treatments should be delivered.

Research should not only be aimed at common mental disorders, but also at bipolar and psychotic disorders.

Although there is already some research on cost effectiveness of Internet-based treatment, this should be expanded to get a full overview of the benefits.

Technological innovations in e-Mental health and Internet-based treatments can contribute considerably to the further development of these treatments

- Research on the use of mobile applications may advance the field considerably.

- Ecological Momentary Assessment (EMA) is a promising new field that may have a large impact on Internet-based treatments but also on the field of psychology and psychiatry in general.

- More research in other new technologies is needed: the development of telepsychiatry, the development of avatars as coaches in psychological interventions, virtual reality, and serious gaming. of propositions (table 3) for what type of action might be particularly fruitful to substantially advance our understanding of mental disorders and the way psychotherapy might help to reduce the burden.

We have emphasized that psychological models of mental disorders can guide research into psychological and environmental factors that elicit and maintain mental disorders as well as interventions to reduce them. In this respect, 4 areas relevant for psychotherapy research need to be highlighted: (1) psychological interventions for mental disorders should increasingly target specific cognitive dysfunctions and specific mechanism and examine potential endophenotypes in addition to traditional psychopathological and diagnostic targets; (2) component analyses aiming at an optimal identification of core ingredients and the mechanisms of change should be used to improve efficacy and effectiveness of psychotherapy, as well as improved translation to rou- tine care; (3) moderator and mediator analyses should be emphasized; and (4) psychotherapy research needs to broaden in terms of adoption of large-scale public health strategies and treatments that can be applied to more patients in a simpler and cost-effective way.

As we have consistently argued throughout this article, the framework for guiding or organizing this comprehensive and concerted research agenda can, in our view, only be provided by the psychological sciences.

\section{Disclosure Statement}

The authors declare that there is no conflict of interest concerning this paper.

\section{References}

American Psychiatric Association (APA): Diagnostic and Statistical Manual of Mental Disorders, Text Revision (4th ed). Arlington, APA, 2000.

American Psychiatric Association (APA): Diagnostic and Statistical Manual of Mental Disorders (5th ed). Arlington, APA, 2013
Andrews G, Charney DS, Sirovatka PJ, Regier DA: Stressinduced and fear circuitry disorders: refining the research agenda for DSM-V. Arlington, APA, 2009.

Arch J, Craske M: First-line treatment: a critical appraisal of cognitive behavioral therapy developments and alternatives. Psychiatr Clin North Am 2009;32:525-547.
Beesdo-Baum K, Knappe S: Developmental epidemiology of anxiety disorders. Child Adolesc Psychiatr Clin North Am 2012;21:457-478.

Butler AC, Chapman JE, Forman EM, Beck AT: The empirical status of cognitive-behavioral therapy: a review of meta-analyses. Clin Psychol Rev 2006;26:17-31. 
Cacioppo JT: Psychology is a hub science. Observer 2007; $20: 5,42$

Craske M: Optimizing exposure therapy for anxiety disorders: an inhibitory learning and inhibitory regulation approach. Verhaltenstherapie 2015:25:134-143.

Crawcour S, Leibing E, Ginzburg D, et al.: Transfer of manualized CBT for social phobia into clinical practice (SOPHO-PRAX): A study protocol for a cluster-randomized controlled trial. Trials 2012;13:70.

David D, Montgomery GH: The scientific status of psychotherapies: a new evaluative framework for evidencebased psychosocial interventions. Clin Psychol Sci Pract 2011;18:89-99.

Domschke K, Deckert J: Genetics of anxiety disorders status quo and quo vadis. Curr Pharm Des 2012;18: 5691-5698.

Ehring T, Watkins ER: Repetitive negative thinking as a transdiagnostic process. Int J Cognit Psychother 2008; 1:192-205.

Emmelkamp PMG, David D, Beckers T, et al.: Advancing psychotherapy and evidence-based psychological in terventions. Int J Methods Psychiatr Res 2014; 23(suppl1):58-91.

Etkin A, Wager TD: Functional neuroimaging of anxiety: a meta-analysis of emotional processing in PTSD, social anxiety disorder, and specific phobia. Am J Psychiatry 2007;164:1476-1488.

Fava GA, Tossani E, Bech P, et al.: Emerging clinical trends and perspectives of comorbid patterns of mental disorders. Int J Methods Psychiatr Res 2014;23(suppl1):92101.

Forand NR, De Rubeis RJ, Amsterdam JD: Combining medication and psychotherapy in the treatment of mental disorders; in Lambert MJ (ed): Bergin and Garfield's handbook of psychotherapy and behaviour change, ed 6. New York, John Wiley \& Sons, 2013, pp 735-775.

Fors EA, Bertheussen GF, Thune I, et al.: Psychosocial interventions as part of breast cancer rehabilitation programs? Results from a systemic review. Psychooncology 2011;20:909-918.

Geller J, Dunn EC: Integrating motivational interviewing and cognitive behavioral therapy in the treatment of eating disorders: tailoring interventions to patient readiness for change. Cog Behav Pract 2011;18:5-15.

Gloster AT, Wittchen H-U, Einsle F, et al.: Psychological treatment for panic disorder with agoraphobia: A randomized controlled trial to examine the role of therapist-guided exposure in situ in CBT. J Consult Clin Psychol 2011;79:406-420.

Gloster AT, Sonntag R, Hoyer J, et al.: Treating treatmentresistant patients with panic disorder and agoraphobia using psychotherapy: a randomized controlled switching trial. Psychother Psychosom 2015;84:100-109.

Goschke T: Dysfunctions of decision-making and cognitive control as transdiagnostic mechanisms of mental disorders: advances, gaps, and needs in current research. Int J Methods Psychiatr Res 2014;23(suppl1):41-57.

Grilo CM, Crosby RD, Wilson GT, Masheb RM: 12-month follow-up of fluoxetine and cognitive behavioral therapy for binge eating disorder. J Consult Clin Psychol 2012;80:1108-1113.

Gulliksson M, Burell G, Vessby B, et al.: Randomized controlled trial of cognitive behavioral therapy vs standard treatment to prevent recurrent cardiovascular events in patients with coronary heart disease: Secondary Prevention In Uppsala Primary Health Care Project (SUPRIM). Arch Intern Med 2011;171:134-140

Gustavsson A, Svensson M, Jacobi F, et al.: Cost of disorders of the brain in Europe 2010. Eur Neuropsychopharmacol 2011;21:718-779.

Haber SN, Rauch SL: Neurocircuitry: a window into the networks underlying neuropsychiatric disease. Neuropsychopharmacology 2010;35: 1-3. ropean Methods Psychiatr Res 2014;23(suppl1):1-14.

Haslam N, Lusher D: The structure of mental health research: networks of influence among psychiatry and clinical psychology journals. Psychol Med 2011;41: 2661-2668.

Hofmann SG, Smits JAJ: Cognitive-behavioral therapy for adult anxiety disorders: a meta-analysis of randomized placebo-controlled trials. J Clin Psychiatry 2008;69: 621-632.

Hofmann SG, Sawyer AT, Fang A, Asnaani A: Emotion dysregulation model of mood and anxiety disorders. Depress Anxiety 2012;29:409-416.

Hutton P, Taylor PJ: Cognitive behavioural therapy for psychosis prevention: a systematic review and metaanalyses. Psychol Med 2013;22:1-20.

Jones C, Hacker D, Cormac I, et al.: Cognitive behaviour therapy versus other psychosocial treatments for schizophrenia. Cochrane Database Syst Rev 2012a;4:CD008712.

Jones S, Mulligan LD, Law H, et al.: A randomized controlled trial of recovery focused CBT for individuals with early bipolar disorder. BMC Psychiatry 2012b;12:204.

Kashikar-Zuck S, Sil S, Lynch-Jordan AM, et al.: Changes in pain coping, catastrophizing, and coping efficacy after cognitive-behavioral therapy in children and adolescents with juvenile fibromyalgia. J Pain 2012;14:492-501.

Kessler RC, Berglund P, Demler O, et al.: Lifetime prevalence and age-of-onset distributions of DSM-IV disorders in the National Comorbidity Survey Replication. Arch Gen Psychiatry 2005;62:593-602.

Kircher T, Arolt V, Jansen A, et al.: Effect of cognitive-behavioural therapy on neural correlates of fear conditioning in panic disorder. Biol Psychiatry 2012;73:93-101.

Kirsch I: Changing Expectations. A Key to Effective Psychotherapy. Pacific, Brooks/Cole Publishing Co., 1990.

Koob GF, Le Moal M: Drug addiction, dysregulation of reward, and allostasis. Neuropsychopharmacology 2001; 24:97-129.

Kraemer HC: A mediator effect size in randomized clinical trials. Int J Methods Psychiatr Res 2014;23:401-410.

Lueken U, Kruschwitz JD, Muehlhan M, et al.: How specific is specific phobia? Different neural response patterns in two subtypes of specific phobia. Neuroimage 2011;56:363-372.

Merry SN, Stasiak K, Shepherd M, et al.: The effectiveness of SPARX, a computerised self help intervention for adolescents seeking help for depression: randomised controlled non-inferiority trial. BMJ 2012;344:e2598.

Meyerbröker K, Morina N, Kerkhof GA, Emmelkamp PMG: Virtual reality exposure therapy does not provide any additional value in agoraphobic patients: a randomized controlled trial. Psychother Psychosom 2013;82:170-176.

National Advisory Mental Health Council (NAMHC): Transformative neurodevelopmental research in mental illness. Washington, NIMH, 2008.

Neshat-Doost HT, Dalgleish T, Yule W, et al.: Enhancing autobiographical memory specificity through cognitive training: an intervention for depression translated from basic science. Clin Psychol Sci 2013;1:84-92.

Pellmar TC, Eisenberg L: Bridging Disciplines in the Brain, Behavioral, and Clinical Sciences. Washington DC, IOM/The National Academies Press, 2000.

Raes F, Williams JMG, Hermans D: Reducing cognitive vulnerability to depression: a preliminary evaluation of MEmory Specificity Training (MEST) in inpatients with depressive complaints. J Behav Ther Exp Psychiatry 2009;40:24-38.

ROAMER (A Roadmap for Mental Health Research in Europe). www.roamer-mh.org (accessed May 1, 2013).

Robbins TW, Gillan CM, Smith DG, et al.: Neurocognitive endophenotypes of impulsivity and compulsivity: towards dimensional psychiatry. Trends Cogn Sci 2011; 16:81-91.
Roth AD, Fonagy P: What works for whom? A critical review of psychotherapy research. New York, Guilford, 1996.

Sava F, Yates B, Lupu V, et al.: Cost-effectiveness and costutility of cognitive therapy, rational emotive behaviour therapy, and fluoxetine (Prozac) in treating depression: a randomized clinical trial. J Clin Psychol 2009; 65:36-52.

Schoenmakers TM, de Bruin M, Lux IFM, et al.: Clinical effectiveness of attentional bias modification training in abstinent alcoholic patients. Drug Alcohol Depend 2010;109:30-36.

Schumann G, Binder EB, Holte A, et al.: Stratiefied medicine for mental disorders. Eur Neuropsychopharmacol 2014;24:5-50.

Siegle GJ, Thompson WK, Collier A, et al.: Towards clinically useful neuroimaging in depression treatment: Is subgenual cingulate activity robustly prognostic for depression outcome in cognitive therapy across studies, scanners, and patient characteristics? Arch Gen Psychiatry 2013;69:913-924.

Shin LM, Liberzon I: The neurocircuitry of fear, stress, and anxiety disorders. Neuropsychopharmacology 2010; 35:169-191.

Skuse DH: Endophenotypes and child psychiatry. Br J Psychiatry 2001;178:395-396

Teachman B: Mechanisms of change in CBT; in Emmelkamp PMG, Ehring T (eds): The Wiley Blackwell International Handbook of Anxiety Disorders, Vol. I. Oxford, Wiley-Blackwell, 2014

Van der Feltz-Cornelis CM, Adèr HJ: Randomization in psychiatric intervention research in the general practice setting. Int J Methods Psychiatr Res 2000;9:134-142.

Van der Gaag M, Nieman DH, Rietdijk J, et al.: Cognitive behavioral therapy for subjects at ultrahigh risk for developing psychosis: A randomized controlled clinical trial. Schizophr Bull 2012;38:1180-1188.

Van der Oord S, Ponsioen A, Geurts H, et al.: A pilot study of the efficacy of a computerized executive functioning remediation training with game elements for children with ADHD in an outpatient setting: outcome on parent and teacher-rated executive functioning and ADHD behavior. J Atten Disord 2014;18:699-712.

Wampold BE: The great psychotherapy debate: Models, methods and findings. Mahwah, Erlbaum, 2001.

Wang PS, Berglund PA, Olfson M, et al.: Failure and delay in initial treatment contact after first onset of mental disorders in the National Comorbidity Survey Replication (NCS-R). Arch Gen Psychiatry 2005;62:603-613.

Williams JMG, Barnhofer T, Crane C, et al.: Autobiographical memory specificity and emotional disorder. Psychol Bull 2007;133:122-148.

Wittchen H-U: Editorial. Int J Methods Psychiatr Res 2014;23(suppl1):v-vi.

Wittchen H-U, Hoyer J: Was ist klinische Psychologie? Definitionen, Konzepte und Modelle; in dies (eds): Klinische Psychologie und Psychotherapie, ed 2. Berlin, Springer, 2011, pp 4-25.

Wittchen H-U, Knappe S, Andersson G, et al.: The need for a behavioural science focus in research on mental health and mental disorders. Int J Methods Psychiatr Res 2014a;23(suppl1):28-40.

Wittchen H-U, Knappe S, Schumann G: The psychological perspective on mental health and mental disorder research. Int J Methods Psychiatr Res 2014b;23(suppl1): $15-27$.

Wittchen H-U, Jacobi F, Rehm J, et al.: The size and burden of mental disorders and other disorders of the brain in Europe 2010. Eur Neuropsychopharmacol 2011;21:655-679.

World Health Organization (WHO): Tenth Revision of the International Classification of Diseases Chapter V (F): Mental and Behavioral Disorders (Including Disorders of Psychological Development). Clinical Descriptions and Diagnostic Guidelines. Geneva, WHO, 1991. 